\title{
Increased skeletal muscle ceramide level in men at risk of developing type 2 diabetes
}

\author{
M. Straczkowski • I. Kowalska • M. Baranowski • \\ A. Nikolajuk • E. Otziomek • P. Zabielski • \\ A. Adamska • A. Blachnio • J. Gorski • M. Gorska
}

Received: 8 May 2007 / Accepted: 1 July 2007 / Published online: 28 August 2007

(C) Springer-Verlag 2007

\begin{abstract}
Aims/hypothesis Intramyocellular lipids, including ceramide, a second messenger in the sphingomyelin signalling pathway, might contribute to the development of insulin resistance. The aim of our study was to assess parameters of the skeletal muscle sphingomyelin signalling pathway in men at risk of developing type 2 diabetes.

Methods We studied 12 lean $\left(\mathrm{BMI}<25 \mathrm{~kg} / \mathrm{m}^{2}\right)$ men without a family history of diabetes (control group), 12 lean male offspring of type 2 diabetic patients, and 21 men with overweight or obesity comprising 12 with NGT (obese-NGT) and nine with IGT (obese-IGT). A euglycaemic-hyperinsulinaemic clamp and a biopsy of vastus lateralis muscle were performed. Ceramide, sphingomyelin, sphinganine and sphingosine levels and sphingomyelinase and ceramidase activities were measured in muscle. Muscle diacylglycerol and triacylglycerol levels were estimated in a subgroup of 27 men (comprising men from all the above groups).

Results Compared with the control group, the lean offspring of diabetic patients and the men with overweight or obesity showed lower insulin sensitivity (all $p<0.005$ ) and a greater muscle ceramide level (all $p<0.01$ ). The obese-IGT group had lower insulin sensitivity $(p=0.0018)$ and higher muscle ceramide $(p=0.0022)$ than the obese-NGT group. There
\end{abstract}

\footnotetext{
M. Straczkowski $(\square) \cdot$ I. Kowalska $\cdot$ A. Nikolajuk •

E. Otziomek $\cdot$ A. Adamska $\cdot$ M. Gorska

Department of Endocrinology, Diabetology and Internal

Medicine, Medical University of Bialystok,

M.C. Sklodowskiej 24a,

15-276 Bialystok, Poland

e-mail: mstraczkowski@poczta.onet.pl
}

M. Baranowski • P. Zabielski • A. Blachnio · J. Gorski Department of Physiology, Medical University of Bialystok, Bialystok, Poland was lower muscle sphingosine level and alkaline ceramidase activity in offspring of diabetic patients ( $p=0.038$ and $p=0.031$, respectively) and higher sphinganine level in the obese-NGT $(p=0.049)$ and obese-IGT $(p=0.002)$ groups than in the control group. Muscle sphingomyelin was lower $(p=0.0028)$ and neutral sphingomyelinase activity was higher $(p=0.00079)$ in the obese-IGT than in the obeseNGT group. Muscle ceramide was related to insulin sensitivity independently of other muscle lipid fractions. Conclusions/interpretations Ceramide accumulates in muscle of men at risk of developing type 2 diabetes.

Keywords Insulin sensitivity and resistance. Lipid metabolism · Obesity . Weight regulation

\section{Introduction}

A decrease in insulin-stimulated skeletal muscle glucose uptake is an important step in the development of insulin resistance [1]. There is growing evidence for an association of impaired insulin action with intramyocellular lipid accumulation [2]. An increase in intramyocellular lipid level might be observed even in the early stages of the development of insulin resistance, i.e. in lean, non-diabetic participants with a family history of type 2 diabetes [3, 4]. In this group, muscle lipid accumulation was linked to impaired mitochondrial oxidative phosphorylation [5].

One of the intracellular lipid fractions is associated with the sphingomyelin signalling pathway, which is shown in Fig. 1 [6-8]. Ceramide, a second messenger in this pathway, is generated during the hydrolysis of plasma membrane sphingomyelin by the enzyme neutral sphingomyelinase or the hydrolysis of lysosomal and endosomal sphingomyelin by acid sphingomyelinase [9]. Some cer- 
Fig. 1 Scheme of sphingomyelin signalling pathway. ${ }^{\text {a Vari- }}$ ables that were analysed in muscle in the present study. SPT, Serine palmitoyltransferase; S1P, sphingosine-1-phosphate

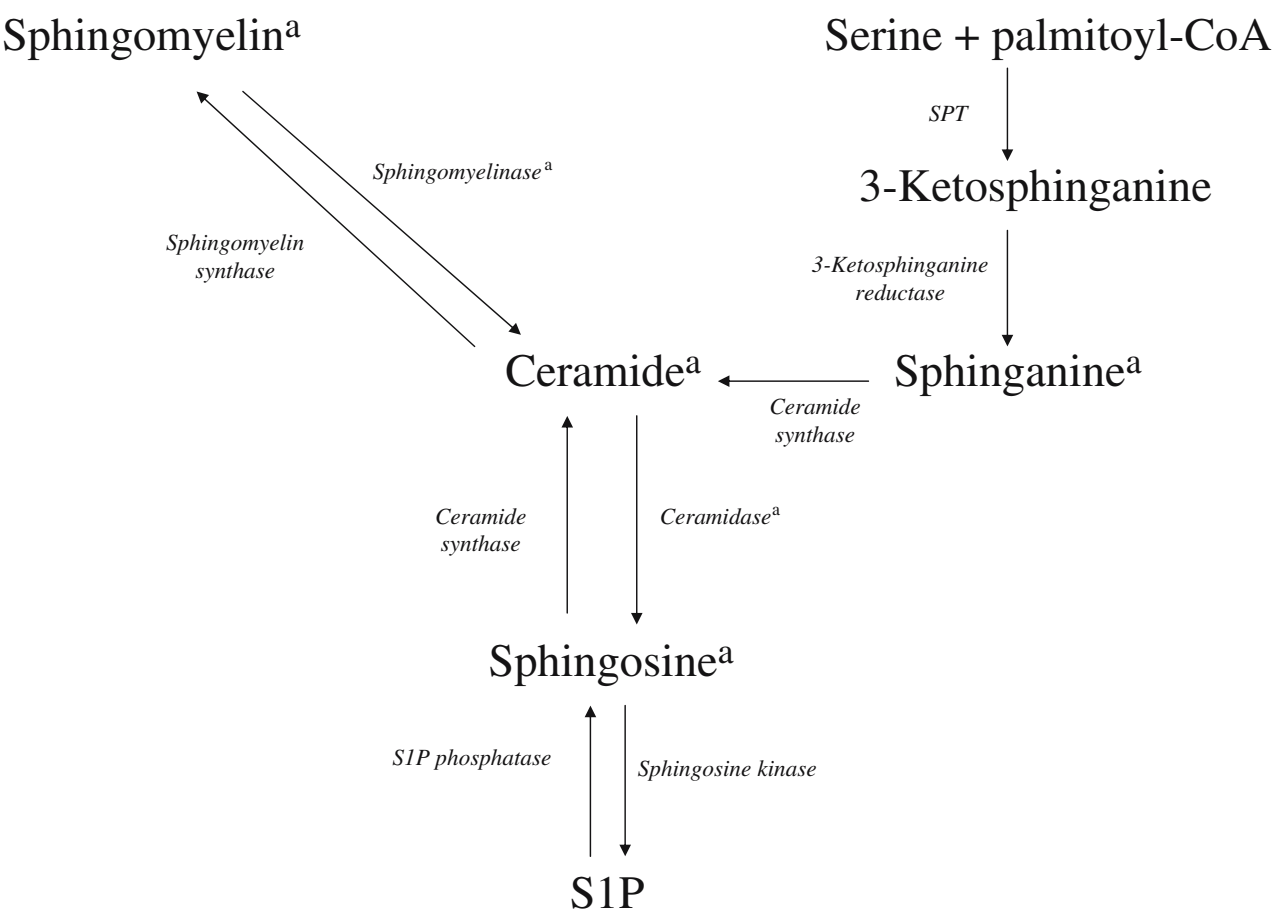

amide is also synthesised in a de novo synthesis pathway [10] from serine and palmitoyl-CoA in which the key intermediate, indicating the activity of this process, is sphinganine. The degradation of ceramide is catalysed by neutral and alkaline ceramidase and the products of this reaction are sphingosine and long-chain fatty acids [11].

Tissue ceramide accumulation might contribute to the development of insulin resistance [12]. Experimental data indicate that ceramide might inhibit insulin signalling at the level of protein kinase B (PKB)/Akt [13]. It blocks insulin stimulation of this kinase by two independent mechanisms, i.e. translocation of PKB to the plasma membrane and stimulation of PKB dephosphorylation by protein phosphatase 2A [14]. Overproduction of acid ceramidase, an enzyme-degrading ceramide, prevented the inhibitory effects of saturated fatty acids on insulin signalling [15]. In mice, deficiency of stearoyl-CoA desaturase 1 (SCD1), an enzyme involved in the regulation of lipid metabolism, led to an improvement in insulin signalling [16] and to a decrease in ceramide synthesis [17]. Although opposite findings regarding the role of SCD1 in modulating insulin action have been reported, an increase in muscle ceramide level also led to inhibition of insulin signalling and glucose uptake [18]. Similarly, administration of ciliary neurotrophic factor prevented the development of lipid-induced insulin resistance and tissue ceramide accumulation [19]. There is also evidence that ceramide-associated insulin resistance is linked to enhanced apoptosis in skeletal muscle myotubes [20].

In line with the above findings, we demonstrated an inverse correlation between muscle ceramide and whole- body insulin sensitivity in humans [21]. Another group reported an increase in muscle ceramide in obese, insulinresistant individuals [22]. In humans, muscle ceramide level is increased by acute physical exercise [23] and decreased by endurance training [24]. No data are available regarding the sphingomyelin signalling pathway in muscles of lean offspring of type 2 diabetic patients and in participants in the prediabetic state, i.e. in obese participants with IGT.

Insulin resistance is also associated with other muscle lipid fractions, such as long-chain fatty acyl-CoA [25], diacylglycerol $[26,27]$ and triacylglycerol $[2,4]$. The interrelationships between these lipid fractions and ceramides in inducing insulin resistance remain unknown.

The aim of the present study was to assess the parameters of the skeletal muscle sphingomyelin signalling pathway in different groups of participants at risk of developing type 2 diabetes, i.e. in lean offspring of type 2 diabetic patients and in obese men with NGT and with IGT.

\section{Methods}

Participants

The study groups consisted of 12 lean (BMI $<25 \mathrm{~kg} / \mathrm{m}$ ) healthy men without a family history of diabetes (control group), 12 lean, healthy male offspring of type 2 diabetic patients, and 21 men with overweight or obesity; of the last group, 12 had NGT (obese-NGT) and nine had IGT (obeseIGT). In the offspring group, only participants with a strong 
family history were recruited, i.e. men having both parents with type 2 diabetes or one parent and one first-degree (brother or sister) or second-degree (grandparent) relative with type 2 diabetes. None of the participants had ischaemic heart disease, unstable hypertension, peripheral vascular disease, an infection or any other serious medical problem; all were non-smokers and none was taking drugs known to affect carbohydrate or lipid metabolism. Glucose tolerance status was estimated on the basis of an OGTT according to WHO criteria. All analyses were performed after an overnight fast. The study protocol was approved by the Ethics Committee of the Medical University, Bialystok. All participants gave written informed consent before entering the study.

\section{Measurements}

Anthropometric and biochemical measurements were performed as previously described [21]. Insulin sensitivity was evaluated with the euglycaemic-hyperinsulinaemic clamp technique, according to DeFronzo et al. [28] as described in [21]. The rate of whole-body glucose uptake ( $M$ value) was calculated as the mean glucose infusion rate from 80 to $120 \mathrm{~min}$, corrected for glucose space and normalised per $\mathrm{kg}$ fat-free mass.

Fasting blood samples were also taken from the antecubital vein before the beginning of the clamp. Serum and plasma samples were frozen at $-20^{\circ} \mathrm{C}$ until analysis for serum insulin, lipids and plasma NEFA.

Before the clamp, a percutaneous biopsy of vastus lateralis muscle was performed with a skeletal muscle biopsy needle $4.5 \mathrm{~mm}$ in diameter (Popper and Sons, New Hyde Park, NY, USA), as described in [21]. The biopsy was taken about $15 \mathrm{~cm}$ above the patella, using local anaesthesia with $1 \%$ lidocaine, after making a small skin incision (about $1 \mathrm{~cm}$ ). Muscles were frozen immediately in liquid nitrogen and were kept at $-70^{\circ} \mathrm{C}$ until analysis. To avoid contamination of extracellular fat, muscle fibres were isolated and lyophilised in liquid nitrogen before analyses.

Analysis of sphingomyelin signalling pathway in muscle Lipids were extracted according to the method of Folch et al. [29] and were then assayed as described in $[8,21]$. Briefly, ceramides and sphingomyelins were separated by thin-layer chromatography on silica gel plates (Kieselgel 60, $0.22 \mathrm{~mm}$; Merck, Darmstadt, Germany). Standards of ceramide (SigmaAldrich, St Louis, MO, USA) and sphingomyelin (SigmaAldrich) were run together with the samples. After separation, fatty acids, together with methylpentadecanoic acid (SigmaAldrich), used as an internal standard, were transmethylated in the presence of $1 \mathrm{ml}$ of $14 \%$ boron fluoride in methanol at $100^{\circ} \mathrm{C}$ for $90 \mathrm{~min}[30]$. The methyl esters were dissolved in $40 \mu \mathrm{l}$ of hexane and analysed by gas-liquid chromatography.
Total ceramide and sphingomyelin level was estimated as the sum of the fatty acid levels of the assessed fraction and was expressed in $\mathrm{nmol} / \mathrm{g}$ tissue $[8,21]$.

Muscle activities of membrane $\mathrm{Mg}^{2+}$-dependent neutral sphingomyelinase and lysosomal $\mathrm{Zn}^{2+}$-independent acid sphingomyelinase were measured according to Liu et al. [31], with the use of a radiolabelled substrate, $N$-cholinemethyl- $\left[{ }^{14} \mathrm{C}\right]$ sphingomyelin (PerkinElmer Life Sciences, Boston, MA, USA), as in our previous studies $[8,21]$. The reaction product, $N$-choline-methyl- $\left[{ }^{14} \mathrm{C}\right]$ phosphocholine, was mixed with $5 \mathrm{ml}$ of scintillation solution for radioactivity counting.

The activities of neutral and alkaline ceramidases in muscle were estimated with a radioisotopic method described by Nikolova-Karakashian et al. [32] using a mixture of substrate containing $1 \mathrm{nmol} \mathrm{N}$-palmitoyl-[1- $\left.{ }^{14} \mathrm{C}\right]$ sphingosine (Moravek Biochemicals, Brea, CA, USA) and 49 nmol unlabelled ceramide (Sigma-Aldrich).

Muscle sphingosine and sphinganine were identified and quantified by HPLC, as reported in detail elsewhere [21, 33].

Analysis of other lipid fractions in muscle Additionally, in a subgroup of 27 men with tissue still available (eight control participants, seven offspring of type 2 diabetic patients, seven obese-NGT men and five obese-IGT men), muscle levels of diacylglycerol and triacylglycerol were measured. These fractions were extracted by thin-layer chromatography [30] and individual fatty acids were analysed by gas-liquid chromatography. Total diacylglycerol and triacylglycerol levels were calculated as the sum of individual fatty acid levels [34].

Other analytical procedures Plasma glucose was measured immediately by the enzymatic method using the glucose analyser YSI 2300 STAT Plus (YSI Inc., Yellow Springs, OH, USA). Serum insulin was measured with a monoclonal immunoradiometric assay (Medgenix Diagnostics, Fleunes, Belgium). Serum total cholesterol, HDL-cholesterol and triacylglycerol were assessed by enzymatic methods (Cormay, Warsaw, Poland) and LDL-cholesterol was calculated with Friedewald's formula. Plasma NEFA were measured by a colorimetric method [35].

\section{Statistical analysis}

Statistical calculations were performed with the Statistica 7.0 program (StatSoft, Krakow, Poland). Differences between groups were evaluated with the Mann-Whitney $U$ test. Offspring, obese-NGT and obese-IGT groups were analysed in comparison with the control group and additional comparisons were made between the obese-NGT and obese-IGT groups. To adjust for differences in age and anthropometry, 
multiple regression analysis with subsequent analysis of residuals was used. Relationships between variables were analysed using Spearman's rank correlation $r$ and multiple regression. Significance was accepted at $p<0.05$.

\section{Results}

Clinical characteristics of the studied groups are shown in Table 1. Compared with the control group, insulin sensitivity was lower in the offspring of type 2 diabetic men $(-27.19 \%$, $p=0.0047)$ obese-NGT men $(-31.99 \%, p=0.0047)$ and obese-IGT men $(-69.81 \%, p=0.00012)$. The obese-IGT group had also lower insulin sensitivity than the obeseNGT group $(p=0.0018)$.

Differences in muscle ceramide between the groups paralleled the differences in insulin sensitivity. Muscle ceramide level was greater in the offspring group $(+49.91 \%$, $p=0.0094)$, the obese-NGT group $(+81.15 \%, p=0.0056)$ and the obese-IGT group $(+199.02 \%, p=0.00012)$ than in the control group and greater in the obese-IGT than in the obeseNGT group $(p=0.0022)$ (Fig. 2). Muscle sphingomyelin level was lower in the obese-IGT than in the obese-NGT group $(p=0.0028)$ and there were no other differences in muscle sphingomyelin between groups (Fig. 2).

No significant differences between the groups were observed in the percentage of saturated (myristic, palmitic, stearic, arachidic and behenic acid), monounsaturated (palmitoleic, oleic and nervonic acid) and polyunsaturated fatty acids (linoleic, linolenic, arachidonic, eicosapentaenoic and docosahexaenoic acid) (Fig. 2) and in the percentage of each particular fatty acid analysed individually as ceramide and sphingomyelin moieties.

Activity of neutral sphingomyelinase was markedly higher in muscles of the obese-IGT men in comparison with the control group $(p=0.00052)$ and the obese-NGT group $(p=0.00078)$. Muscle acid sphingomyelinase activity did not differ markedly between groups, although the difference between the obese-IGT and control groups approached significance $(p=0.089)$ (Table 2).

We found a lower muscle sphingosine level in the offspring group $(p=0.038)$ and a higher level in obese-IGT men $(p=0.031)$ in comparison with the control group. Muscle alkaline ceramidase activity was lower in the offspring group than in the control group $(p=0.031)$. The differences in muscle neutral ceramidase activity between all the studied groups were not significant. We observed a higher muscle sphinganine level in the obese-NGT $(p=$ $0.049)$ and obese-IGT $(p=0.002)$ groups than in the controls (Table 2).

The two groups of obese men were slightly, although nonsignificantly, different in age and anthropometric measurements. However, all the differences found between these groups, i.e. in $M$ value, muscle ceramide, sphingomyelin and neutral sphingomyelinase, were still present after adjustment for age, BMI or waist circumference (all $p<0.05$ ).

In the subgroup of 27 men, there were no differences in muscle diacylglycerol and triacylglycerol between the obeseIGT and obese-NGT groups and between the control and offspring groups. Both obese groups had a higher muscle diacylglycerol level (obese-NGT, $p=0.049$; obese-IGT, $p=$ 0.019 ) and higher triacylglycerol level (obese-NGT, $p=0.02$;

Table 1 Clinical characteristics

\begin{tabular}{|c|c|c|c|c|}
\hline & $\begin{array}{l}\text { Control } \\
(n=12)\end{array}$ & $\begin{array}{l}\text { Offspring }^{\mathrm{a}} \\
(n=12)\end{array}$ & $\begin{array}{l}\text { Obese-NGT } \\
(n=12)\end{array}$ & $\begin{array}{l}\text { Obese-IGT } \\
(n=9)\end{array}$ \\
\hline Age (years) & $28.17 \pm 7.07$ & $27.75 \pm 6.06$ & $28.67 \pm 7.14$ & $34.78 \pm 7.33$ \\
\hline BMI $\left(\mathrm{kg} / \mathrm{m}^{2}\right)$ & $22.74 \pm 1.51$ & $22.33 \pm 2.34$ & $31.66 \pm 3.84^{*}$ & $33.27 \pm 4.70^{*}$ \\
\hline Waist circumference $(\mathrm{cm})$ & $80.46 \pm 7.02$ & $78.04 \pm 8.27$ & $99.33 \pm 9.82 *$ & $106.00 \pm 6.76^{*}$ \\
\hline Body fat $(\%)$ & $16.74 \pm 4.01$ & $14.46 \pm 4.46$ & $30.87 \pm 6.23^{*}$ & $35.76 \pm 6.41 *$ \\
\hline Fasting glucose $(\mathrm{mmol} / \mathrm{l})$ & $4.78 \pm 0.47$ & $4.81 \pm 0.45$ & $5.10 \pm 0.35$ & $5.62 \pm 0.47 * * *$ \\
\hline Postload glucose (mmol/l) & $4.49 \pm 1.21$ & $4.96 \pm 1.51$ & $5.20 \pm 1.19$ & $8.69 \pm 0.81^{*, * *}$ \\
\hline Fasting insulin (pmol/l) & $70.02 \pm 37.69$ & $59.79 \pm 21.39$ & $95.19 \pm 56.60$ & $162.23 \pm 101.13^{*}$ \\
\hline Postload insulin (pmol/1) & $147.6 \pm 126.5$ & $177.2 \pm 139.5$ & $244.9 \pm 154.1$ & $760.2 \pm 288.4^{* * * *}$ \\
\hline$M\left(\mu \mathrm{mol} \mathrm{min}{ }^{-1} \mathrm{~kg}\right.$ fat-free mass $\left.{ }^{-1}\right)$ & $64.79 \pm 14.43$ & $47.17 \pm 12.57^{*}$ & $44.06 \pm 13.95^{*}$ & $19.56 \pm 9.64 * * *$ \\
\hline Plasma NEFA (mmol/l) & $0.44 \pm 0.16$ & $0.43 \pm 0.09$ & $0.59 \pm 0.26$ & $0.75 \pm 0.21^{*}$ \\
\hline Total cholesterol $(\mathrm{mmol} / \mathrm{l})$ & $4.36 \pm 0.51$ & $4.81 \pm 0.64$ & $5.00 \pm 1.17$ & $5.47 \pm 1.57^{*}$ \\
\hline Triacylglycerol (mmol/l) & $0.88 \pm 0.26$ & $0.92 \pm 0.49$ & $1.77 \pm 1.11^{*}$ & $2.14 \pm 1.35^{*}$ \\
\hline HDL-cholesterol (mmol/1) & $1.35 \pm 0.33$ & $1.55 \pm 0.24$ & $1.23 \pm 0.36$ & $1.02 \pm 0.11^{*}$ \\
\hline LDL-cholesterol (mmol/l) & $2.61 \pm 0.60$ & $2.83 \pm 0.52$ & $3.05 \pm 1.07$ & $3.48 \pm 1.09^{*}$ \\
\hline
\end{tabular}

Data are mean $\pm \mathrm{SD}$

$M$ Rate of whole-body glucose uptake

${ }^{*} p<0.05$ in offspring, obese-NGT or obese-IGT group in comparison with controls

$* * p<0.05$ in obese-IGT vs obese-NGT group

${ }^{\mathrm{a}}$ Offspring of type 2 diabetic patients 


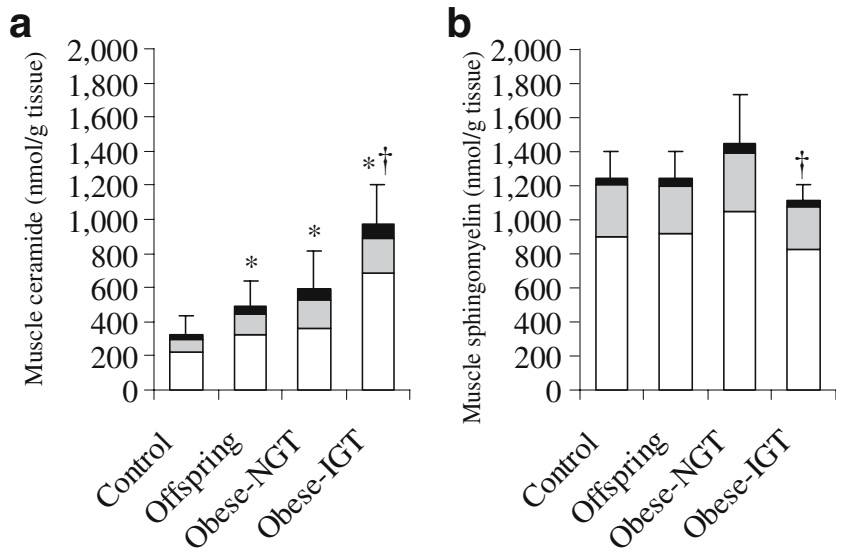

Fig. 2 Total ceramide (a) and sphingomyelin (b) concentrations in muscle. Open bar, saturated fatty acids; grey bar, monounsaturated fatty acids; closed bar, polyunsaturated fatty acids. ${ }^{*} p<0.05$ in offspring, obese-NGT and obese-IGT groups vs control group. $\dagger p<$ 0.05 in obese-IGT vs obese-NGT group

obese-IGT, $p=0.013$ ) in comparison with the controls (Table 2). The study groups did not differ in the percentages of individual fatty acids in diacylglycerol and triacylglycerol.

As in our previous study, muscle ceramide (Fig. 3), sphinganine and sphingosine were inversely related to insulin sensitivity $(r=-0.61, p=0.000007 ; r=-0.49, p=0.0006$; and $r=-0.33, p=0.03$ respectively). After the exclusion of the obese-IGT group from the analysis, associations of ceramide and sphinganine with $M$ value were still present $(r=-0.37$, $p=0.025$ and $r=-0.36, p=0.032$ respectively). Insulin sensitivity was also inversely related to muscle neutral ceramidase activity $(r=-0.37, p=0.02)$. In the entire group, postload glucose was positively correlated with neutral acid sphingomyelinase activity $(r=0.51, p=0.00052)$ and acid sphingomyelinase activity $(r=0.54, p=0.00018)$.

We found that muscle diacylglycerol and triacylglycerol were both negatively related to insulin sensitivity $(r=-0.53$, $p=0.0048$ and $r=-0.52, p=0.0059$, respectively) and that they were not related to muscle ceramide. In the multiple regression analysis, ceramide predicted insulin resistance independently of diacylglycerol (adjusted $\beta=-0.58, p=$ 0.00028 ) and triacylglycerol (adjusted $\beta=-0.56, p=$ $0.00069)$ and both diacylglycerol and triacylglycerol predicted insulin resistance independently of ceramide (adjusted $\beta=-0.38, p=0.0099$ and $\beta=-0.34, p=0.028$, respectively).

\section{Discussion}

In the present study, we observed an increase in muscle ceramide level in men at risk of developing type 2 diabetes. The mechanism of muscle ceramide accumulation might have differed in the different groups of participants. The greater muscle ceramide level in the offspring of type 2 diabetic patients in comparison with the control group was accompanied by lower muscle alkaline ceramidase activity and lower muscle sphingosine level. These findings suggest that the reason for ceramide accumulation might be an impairment in its degradation. So far, no data are available to compare with our results regarding the impact of family history of type 2 diabetes on ceramide metabolism in muscle. Experimental studies indicate that inhibition of ceramide degradation might have a profound effect on steady-state ceramide levels in tissues and might augment ceramide-associated insulin resistance [36].

The higher muscle ceramide level in the obese individuals observed in our study is in agreement with the data of Adams et al. [22], although Serlie et al. [37] did not observe a difference between lean and overweight/obese individuals. In our study, the concurrent increase in muscle sphinganine might indicate that ceramide accumulation in obesity might be due to an increased rate of its production in the de novo synthesis pathway. The increase in serum NEFA reached significance only in the obese-IGT group in comparison with control group; however, one might hypothesise that

Table 2 Muscle parameters of sphingomyelin signalling pathway and muscle diacylglycerol and triacylglycerol concentrations

\begin{tabular}{|c|c|c|c|c|}
\hline & $\begin{array}{l}\text { Control } \\
(n=12)\end{array}$ & $\begin{array}{l}\text { Offspring }^{\mathrm{a}} \\
(n=12)\end{array}$ & $\begin{array}{l}\text { Obese-NGT } \\
(n=12)\end{array}$ & $\begin{array}{l}\text { Obese-IGT } \\
(n=9)\end{array}$ \\
\hline Neutral sphingomyelinase (nmol ceramide $\mathrm{h}^{-1} \mathrm{mg}$ protein ${ }^{-1}$ ) & $0.69 \pm 0.15$ & $0.69 \pm 0.21$ & $0.71 \pm 0.21$ & $0.99 \pm 0.09^{* * * *}$ \\
\hline Acid sphingomyelinase (nmol ceramide $\mathrm{h}^{-1} \mathrm{mg}$ protein ${ }^{-1}$ ) & $3.12 \pm 0.51$ & $3.24 \pm 0.89$ & $3.35 \pm 0.96$ & $3.62 \pm 0.60$ \\
\hline Sphingosine (nmol/g tissue) & $0.91 \pm 0.10$ & $0.80 \pm 0.12 *$ & $0.98 \pm 0.17$ & $1.03 \pm 0.10^{*}$ \\
\hline Sphinganine (nmol/g tissue) & $0.28 \pm 0.05$ & $0.29 \pm 0.07$ & $0.36 \pm 0.09 *$ & $0.38 \pm 0.07 *$ \\
\hline Alkaline ceramidase (nmol sphingosine $\mathrm{h}^{-1} \mathrm{mg}$ protein ${ }^{-1}$ ) & $0.26 \pm 0.06$ & $0.20 \pm 0.06^{*}$ & $0.31 \pm 0.06$ & $0.31 \pm 0.08$ \\
\hline Neutral ceramidase (nmol sphingosine $\mathrm{h}^{-1} \mathrm{mg}_{\text {protein }}{ }^{-1}$ ) & $0.28 \pm 0.09$ & $0.24 \pm 0.05$ & $0.34 \pm 0.06$ & $0.34 \pm 0.08$ \\
\hline Diacylglycerol (nmol/g tissue) & $2127.7 \pm 701.6$ & $2271.8 \pm 613.9$ & $2973.5 \pm 766.4^{*}$ & $3220.1 \pm 643.2 *$ \\
\hline Triacylglycerol $(\mu \mathrm{mol} / \mathrm{g}$ tissue $)$ & $98.99 \pm 40.63$ & $108.30 \pm 51.56$ & $160.10 \pm 38.53^{*}$ & $165.22 \pm 25.53 *$ \\
\hline
\end{tabular}

Data are mean $\pm \mathrm{SD}$

${ }^{*} p<0.05$ in offspring, obese-NGT or obese-IGT group vs controls

$* * p<0.05$ in obese-IGT vs obese-NGT group

${ }^{\text {a }}$ Offspring of type 2 diabetic patients 


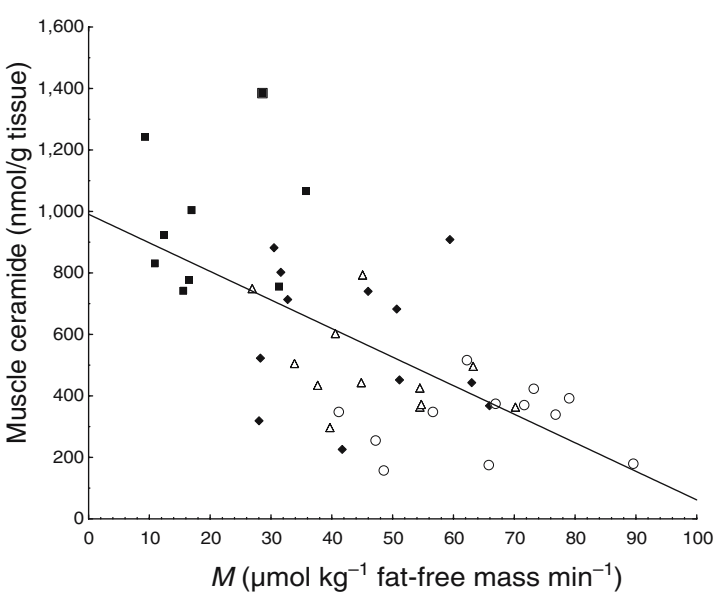

Fig. 3 Relationship between muscle ceramide concentration and insulin sensitivity. Circles, controls; triangles, offspring; diamonds, obese-NGT; squares, obese IGT. $r=-0.61, p=0.000007$

enhanced de novo synthesis is dependent on NEFA, as exposure to long-chain saturated fatty acids promotes ceramide accumulation [38].

The greater neutral sphingomyelinase activity and lower muscle sphingomyelin level in the obese-IGT group in comparison to the obese-NGT group suggest that the larger increase in muscle ceramide level observed in this group might have resulted from greater sphingomyelin hydrolysis. Although the cross-sectional design of our study does not allow us to establish causality, one might hypothesise that this is a secondary phenomenon. If sphingomyelin hydrolysis was the result of obesity, it should also have been observed in the obese-NGT group, and if it was genetically determined it should have been noted in the offspring group. In previous reports, an increase in ceramide with a concurrent decrease in sphingomyelin was reported in the muscles of rats with streptozotocin-induced diabetes [39] and in the sera of type 2 diabetic patients [40]. In our in vitro experiments with the rat soleus muscle, we observed that the addition of glucose to the incubation medium had no effect on the neutral sphingomyelinase activity (M. Straczkowski and P. Zabielski, unpublished observations). It is therefore unlikely that hyperglycaemia itself stimulates tissue neutral sphingomyelinase activity and one might hypothesise that the observed findings are associated with other factors, so far unknown, related to the prediabetic or diabetic state.

As in our previous study, muscle ceramide was inversely related to insulin sensitivity [21]. Experimental studies revealed that even relatively small changes in the tissue ceramide level are sufficient to antagonise insulin action [36]. Data on the lean offspring of type 2 diabetic patients suggest that defective ceramide degradation is a primary abnormality, which leads to accumulation of ceramide in muscle and the subsequent development of insulin resistance.

Given the facts that studies in muscle culture demonstrate that overproduction of acid ceramidase prevents the inhibitory effects of saturated fatty acids on insulin signalling [15] and that muscle alkaline ceramidase activity was decreased in the offspring group, it was tempting to speculate that ceramidases would be positively associated with insulin action. In fact, we observed the opposite. When the entire group of participants was analysed, muscle neutral ceramidase activity was inversely related to insulin sensitivity and both ceramidases were increased (although non-significantly) in the obese groups. We hypothesised that, in obesity, a slight increase in muscle ceramidase activity is secondary to an increase in ceramide, in order to prevent its further accumulation. In obese people additional factors are present, resulting in an imbalance between ceramide synthesis and degradation and, in consequence, further aggravation of insulin resistance. Direct causality cannot be established on the basis of the present research, and this should be considered as an important limitation of our study. Another limitation is the fact that we performed only static measurements of the muscle activities of enzymes regulating ceramide synthesis and degradation and the muscle levels of ceramide metabolites, and these measurements do not necessarily reflect the changes in the dynamic metabolic processes. The results of the present study might suggest only indirectly which processes lead to muscle ceramide accumulation in the different groups of men at risk of type 2 diabetes. A possible limitation of the present study is also associated with the relatively low number of participants in the studied groups. Larger studies are required to confirm our findings.

As already mentioned, in other studies long-chain fatty acyl-CoA [25] and diacylglycerol [26, 27] were found to be the main muscle mediators of lipid-induced insulin resistance. Exercise training in humans decreased both ceramide and diacylglycerol level in muscle, especially the saturated fatty acid level of both lipid classes [24]. In our study, the relationship between muscle ceramide level and insulin sensitivity was independent of muscle diacylglycerol and triacylglycerol. Similarly, muscle diacylglycerol and triacylglycerol determined insulin resistance independently of muscle ceramide level. Our data indicate that there are multiple possible pathways mediating lipid-induced insulin resistance.

The findings of the present study provide new information about an increase in muscle ceramide in men at risk of type 2 diabetes. Two recent experimental studies identified ceramide as a key mediator of the development of insulin resistance in muscle. In the first of these, incubation of human muscle cells with palmitate resulted in the accumulation of ceramide and diacylglycerol. The development of palmitate-induced insulin resistance was blocked in the presence of inhibitors of de novo ceramide synthesis [41]. In the second study, inhibition of ceramide synthesis prevented the development of insulin resistance in obese rodents [42]. So far, there is a lack of data regarding the effect of specific treatment to decrease tissue 
ceramide on the action of insulin in vivo in humans. Given the findings of our and other studies, it might be useful to examine whether treatment to decrease tissue ceramide level leads to an improvement in insulin action. It would also be interesting to test whether genes encoding the enzymes regulating ceramide metabolism influence the predisposition to type 2 diabetes.

In conclusion, ceramide accumulates in muscle in men at risk of type 2 diabetes. Our data suggest that, in the offspring of the type 2 diabetic patients, this was the result of decreased ceramide degradation, whereas in the obese groups there was increased de novo ceramide synthesis (in both obese-NGT and obese-IGT individuals) and sphingomyelin hydrolysis (in the obese-IGT group).

Acknowledgements This study was supported by grant 3 P05B 179 22 from the Polish State Committee for Scientific Research, grant 350677 from the Medical University of Bialystok, Poland, and by the EU Project Exogenesis, no. LSHM-CT-2004-005272.

Duality of interest The authors declare that there is no duality of interest associated with this manuscript.

\section{References}

1. Vaag A, Henriksen JE, Beck-Nielsen H (1992) Decreased insulin activation of glycogen synthase in skeletal muscles in young nonobese Caucasian first-degree relatives of patients with noninsulin-dependent diabetes mellitus. J Clin Invest 89:782-788

2. Pan DA, Lillioja S, Kriketos AD et al (1997) Skeletal muscle triglyceride levels are inversely related to insulin action. Diabetes 46:983-988

3. Jacob S, Machann J, Rett K et al (1999) Association of increased intramyocellular lipid content with insulin resistance in lean nondiabetic offspring of type 2 diabetic subjects. Diabetes 48:1113-1119

4. Perseghin G, Scifo P, De Cobelli F et al (1999) Intramyocellular triglyceride content is a determinant of in vivo insulin resistance in humans. Diabetes 48:1600-1606

5. Petersen KF, Dufour S, Befroy D, Garcia R, Shulman GI (2004) Impaired mitochondrial activity in the insulin-resistant offspring of patients with type 2 diabetes. N Engl J Med 350:664-671

6. Kolesnick R (2002) The therapeutic potential of modulating the ceramide/sphingomyelin pathway. J Clin Invest 110:3-8

7. Summers SA, Nelson DH (2005) A role for sphingolipids in producing the common features of type 2 diabetes, metabolic syndrome X, and Cushing's syndrome. Diabetes 54:591-602

8. Dobrzyn A, Gorski J (2002) Ceramides and sphingomyelins in skeletal muscles of the rat: content and composition. Am J Physiol 282:E277-E285

9. Kolesnick RN (1987) 1,2-Diacylglycerols but not phorbol esters stimulate sphingomyelin hydrolysis in $\mathrm{GH}_{3}$ pituitary cells. J Biol Chem 262:16759-16762

10. Bose R, Verheij M, Haimovitz-Friedman A, Scotto K, Fuks Z, Kolesnick RN (1995) Ceramide synthase mediates daunorubicininduced apoptosis: an alternative mechanism for generating death signals. Cell 82:405-414

11. Wertz PW, Downing DT (1990) Ceramidase activity in porcine epidermis. FEBS Lett 268:110-112

12. Summers SA (2006) Ceramides in insulin resistance and lipotoxicity. Prog Lipid Res 45:42-72
13. Schmitz-Pfeiffer C, Craig DL, Biden TJ (1999) Ceramide generation is sufficient to account for the inhibition of the insulin-stimulated $\mathrm{PKB}$ pathway in $\mathrm{C} 2 \mathrm{C} 12$ skeletal muscle cells pretreated with palmitate. J Biol Chem 274:24202-24210

14. Stratford S, Hoehn KL, Liu F, Summers SA (2004) Regulation of insulin action by ceramide: dual mechanisms linking ceramide accumulation to the inhibition of akt/protein kinase B. J Biol Chem 279:36608-36615

15. Chavez JA, Holland WL, Bar J, Sandhoff K, Summers SA (2005) Acid ceramidase overexpression prevents the inhibitory effects of saturated fatty acids on insulin signaling. J Biol Chem 280:20148-20153

16. Rahman SM, Dobrzyn A, Dobrzyn P, Lee SH, Miyazaki M, Ntambi JM (2003) Stearoyl-CoA desaturase 1 deficiency elevates insulinsignaling components and down-regulates protein-tyrosine phosphatase 1B in muscle. Proc Natl Acad Sci USA 100:11110-11115

17. Dobrzyn A, Dobrzyn P, Lee SH et al (2005) Stearoyl-CoA desaturase-1 deficiency reduces ceramide synthesis by downregulating serine palmitoyltransferase and increasing beta-oxidation in skeletal muscle. Am J Physiol 288:E599-E607

18. Pinnamanemi SK, Southgate RJ, Febbraio MA, Watt MJ (2006) Stearoyl CoA desaturase 1 is elevated in obesity but protects against fatty acid-induced skeletal muscle insulin resistance in vitro. Diabetologia 49:3027-3037

19. Watt MJ, Hevener A, Lancaster GI, Febbraio MA (2006) Ciliary neurotrophic factor prevents acute lipid-induced insulin resistance by attenuating ceramide accumulation and phosphorylation of c-Jun N-terminal kinase in peripheral tissues. Endocrinology 147:2077-2085

20. Turpin SM, Lancaster GI, Darby I, Febbraio MA, Watt MJ (2006) Apoptosis in skeletal muscle myotubes is induced by ceramides and is positively related to insulin resistance. Am J Physiol 291: E1341-E1350

21. Straczkowski M, Kowalska I, Nikolajuk A et al (2004) Relationship between insulin sensitivity and sphingomyelin signaling pathway in human skeletal muscle. Diabetes 53:1215-1221

22. Adams JM, Pratipanawatr T, Berria R et al (2004) Ceramide content is increased in skeletal muscle from obese insulin-resistant humans. Diabetes 53:25-31

23. Helge JW, Dobrzyn A, Saltin B, Gorski J (2004) Exercise and training effects on ceramide metabolism in human skeletal muscle. Exp Physiol 89:119-127

24. Bruce CR, Thrush AB, Mertz VA et al (2006) Endurance training in obese humans improves glucose tolerance and mitochondrial fatty acid oxidation and alters muscle lipid content. Am J Physiol 291:E99-E107

25. Bandyopadhyay GK, Yu JG, Ofrecio J, Olefsky JM (2006) Increased malonyl-CoA levels in muscle from obese and type 2 diabetic subjects lead to decreased fatty acid oxidation and increased lipogenesis: thiazolidinedione treatment reverses these defects. Diabetes 55:2277-2285

26. Turinsky J, O’Sullivan DM, Bayly BP (1990) 1,2-Diacylglycerol and ceramide levels in insulin resistant tissues of the rat in vivo. J Biol Chem 265:16880-16885

27. Itani SI, Ruderrman NB, Schmieder F, Boden G (2002) Lipidinduced insulin resistance in human muscle is associated with changes in diacylglycerol, protein kinase $\mathrm{C}$, and IkappaB-alpha. Diabetes 51:2005-2011

28. DeFronzo RA, Tobin JD, Andres R (1979) Glucose clamp technique: a method for quantifying insulin secretion and resistance. Am J Physiol 237:E214-E223

29. Folch J, Lees M, Stanley GHS (1957) A simple method for the isolation and purification of total lipids from animal tissues. J Biol Chem 226:497-509

30. Morrison WR, Smith LM (1964) Preparation of fatty acid methyl esters and dimethylacetals from lipids with boron fluoridemethanol. J Lipid Res 5:600-608 
31. Liu B, Hassler DF, Gary KS, Weaver K, Hannun YA (1998) Purification and characterization of a membrane bound neutral $\mathrm{pH}$ optimum magnesium-dependent and phosphatidylserine-stimulated sphingomyelinase from rat brain. J Biol Chem 273:34472-34479

32. Nikolova-Karakashian M, Merrill AH Jr (2000) Ceramidases. Methods Enzymol 311:194-201

33. Dobrzyn A, Gorski J (2002) Effect of acute exercise on the content of free sphinganine and sphingosine in different skeletal muscle types of the rat. Horm Metab Res 34:523-529

34. Van der Vusse GJ, Roemen TH, Reneman RS (1980) Assessment of fatty acids in dog left ventricular myocardium. Biochim Biophys Acta 617:43-49

35. Duncombe WS (1964) The colorimetric microdetermination of nonesterified fatty acids in plasma. Clin Chim Acta 9:122-135

36. Chavez JA, Knotts TA, Wang LP et al (2003) A role for ceramide, but not diacylglycerol, in the antagonism of insulin signal transduction by saturated fatty acids. J Biol Chem 278:1029710303

37. Serlie MJ, Meijer AJ, Groener JE et al (2007) Short-term manipulation of plasma free fatty acids does not change skeletal muscle concentrations of ceramide and glucosylceramide in lean and overweight subjects. J Clin Endocrinol Metab 92:1524 1529

38. Chavez JA, Summers SA (2003) Characterizing the effects of saturated fatty acids on insulin signaling and ceramide and diacylglycerol accumulation in 3T3-L1 adipocytes and $\mathrm{C} 2 \mathrm{C} 12$ myotubes. Arch Biochem Biophys 419:101-109

39. Gorska M, Dobrzyn A, Zendzian-Piotrowska M, Gorski J (2004) Effect of streptozotocin-diabetes on the functioning of the sphingomyelin signaling pathway in skeletal muscles of the rat. Horm Metab Res 36:14-21

40. Gorska M, Baranczuk E, Dobrzyn A (2003) Secretory Zn2+dependent sphingomyelinase activity in the serum of patients with type 2 diabetes is elevated. Horm Metab Res 35:506-507

41. Pickersgill L, Litherland GJ, Greenberg AS, Walker M, Yeaman SJ (2007) Key role for ceramides in mediating insulin resistance in human muscle cells. J Biol Chem 282:12583-12589

42. Holland WL, Brozinick JT, Wang LP et al (2007) Inhibition of ceramide synthesis ameliorates glucocorticoid-, saturated fat-, and obesity-induced insulin resistance. Cell Metab 5:167-179 